\title{
A single point of contact data platform for rehabilitative exercises and equipment
}

\author{
Development of the REHABitation database
}

\author{
Richard Paštěka \\ University of Applied Sciences \\ Technikum Wien, Department of \\ Biomedical, Health \& Sports \\ Engineering \\ Vienna, Austria \\ richard.pasteka@technikum-wien.at
}

\author{
Mathias Forjan \\ University of Applied Sciences \\ Technikum Wien, Department of \\ Biomedical, Health \& Sports \\ Engineering \\ Vienna, Austria \\ mathias.forjan@technikum-wien.at
}

\author{
Veronika David \\ University of Applied Sciences \\ Technikum Wien, Department of \\ Biomedical, Health \& Sports \\ Engineering \\ Wien, Austria \\ veronika.david@technikum-wien.at
}

\begin{abstract}
Considering the increasing burden of costs on health care systems in general, new approaches to rise effectiveness and increase information transfer are to be sought. Telerehabilitation and rehabilitation at home are promising candidates. The REHABitation database was created in order to effectively communicate new concepts and approaches not only to the researchers in the field of rehabilitative medicine and medical staff at the clinic environment, but also to the general public and potential users. By these means also the patients performing home based rehabilitative exercises could benefit from acquired knowledge. To the current point in time the database includes 450 entries which have been accessed by 266 users from 33 states. The REHABitation database provides information as to what innovative or well established exercises/assessments they could include into the rehabilitation process. Information from each sub-database is reachable either separately or via the concise overview given on the main page.
\end{abstract}

\section{CCS CONCEPTS}

- Information systems $\rightarrow$ Data management systems; Users and interactive retrieval; • Applied computing $\rightarrow$ Consumer health; Health informatics;

\section{KEYWORDS}

telemedicine, rehabilitation, home base, database, exercises, medical equipment

\section{ACM Reference Format:}

Richard Paštěka, Mathias Forjan, and Veronika David. 2018. A single point of contact data platform for rehabilitative exercises and equipment: Development of the REHABitation database. In DSAI 2018: 8th International Conference on Software Development and Technologies for Enhancing Accessibility and Fighting Info-exclusion, June 20-22, 2018, Thessaloniki, Greece. ACM, New York, NY, USA, 6 pages. https://doi.org/10.1145/3218585.3218676

Permission to make digital or hard copies of part or all of this work for personal or classroom use is granted without fee provided that copies are not made or distributed for profit or commercial advantage and that copies bear this notice and the full citation on the first page. Copyrights for third-party components of this work must be honored.

For all other uses, contact the owner/author(s).

DSAI 2018, June 20-22, 2018, Thessaloniki, Greece

(C) 2018 Copyright held by the owner/author(s).

ACM ISBN 978-1-4503-6467-6/18/06

https://doi.org/10.1145/3218585.3218676

\section{INTRODUCTION}

Considering the increasing burden of costs on health care systems in general, new approaches to rise effectiveness and increase information transfer are to be sought. Telerehabilitation and rehabilitation at home are promising approaches. These measures can reduce costs and even support patients to re-integrate in daily life activities [12]. Studies have proven that long stay home care patients, undergoing rehabilitation at home, show improved outcomes and cause less costs for the health care system [1]. Taking home based rehabilitation as a potential way to go, corresponding information has to be distributed to affected people. This of course includes the description of prescribed rehabilitative exercises. In many cases this information is transported verbally or in the form of paper copies. Even if this information is completely understood by the patient, the correct performance of these exercises cannot be checked at the home environment, as there are very few tools available. Therefore home-based rehabilitation tools, aligned with well accessible information are a possible way to increase motivation. These tools have to be easily accessible, easy to handle and foremost inexpensive. Such tools shall be connected with the corresponding information of how to use them and which areas of assessment can be trained with them. Another factor for further research and development in the field of telerehabilitation is the increase of active participation in social and cultural as well as occupational areas of life. It has to be a goal of rehabilitative efforts to improve health-related quality of life [11]. During their stay in a rehabilitation clinic patients undergo several and clearly structured treatments and interventions. As soon as they return home this structured organisation is handed over to the patients. Many patients do not perform any of the prescribed exercise when at home, even though the rehabilitation process is actually not completed [2]. Taking the demographic change into account, the need for rehabilitation is rising constantly. This will again lead to a higher financial burden for the healthcare systems [5].

\subsection{Databases For Rehabilitatory Purposes}

The REHABitation database was created in order to effectively communicate new concepts and approaches not only to the researchers in the field of rehabilitative medicine and medical staff at the clinic environment, but also to the general public and potential users. By these means also the patients performing home based rehabilitative exercises could benefit from acquired knowledge. 
The concept of the database together with the description of initial requirements for the database followed by design and usability tests have already been described by [7].

Other available databases focused on the field of rehabilitation usually provide only limited services without payment. Full functionality and all the features can be accessed under the assumption of membership fees. Furthermore, internal links between components of the database are either limited or not present at all. One of promising personal therapist systems is PeThe [8]. Progress of home base rehabilitation of patients can track by a therapist. For this purposes the movements are captured by Microsoft Kinect and stored in Microsoft Cloud Azure. In contrast the REHABitation database allows incorporation of wide variety of tools and equipment such as smart watches, smart phones and Wii balance board.

The uniqueness of the REHABitation database lays in the structure of network links between the database components. Furthermore its open architecture allows multiple user groups to insert tools, new assessments or create new exercises by combining existing components in novel variation. This connection specifically between exercises, assessments, diagnosis, devices and systems indirectly proposes ideas for potential usability of already available equipment for different rehabilitative purposes.

The presented work introduces further advancements of the REHAbitation database. Inclusion of many new exercises and assessments together with their link to the usable equipment shall increase potential output of rehabilitative efforts. Effort was put into increasing usability and accessibility for all user groups by incorporating visually clear and simple straight forward design. In addition the option for viewing details of the selected exercises was introduced containing information and suggestions about how to perform the exercises. The whole process is complemented by media such as video and/or graphical representation of chosen exercise in order to visualized practical usage of the given exercise.

\section{METHOD}

The REHABitation database was developed over the course of the project REHABitation. Since its introduction the database underwent a series of development changes with the aim of increasing potential positive impact it might have especially on home-based rehabilitation process.

The behaviour of users interacting with the REHABitation web based application has been continuously monitored using Google Analytics Solutions [3] and the features of the database modified accordingly. Selected markers which influenced development of the database are: the way user accessed the database and associated web-pages split into three categories 1) referral- user was redirected from different web page 2) organic - access following results from search engine 3) social - page was reached based on social media reference, time spend on the specific web-site and technologies used in order to reach the database (mobile devices and personal computers). Following features has been developed and implemented:

- Detailed view of each exercise entry in the database containing additional specific information for the given exercise

- Concise description and tips on how the exercise should be performed
- Possibility of including graphical representation of the exercise in the form of image

- Embedded video recording from external sources showing exemplary performance of the exercise

- Visually clear, easy to read and graphically concise design

The REHABitation database is using LAMP stack open source web development platform as a building stone of the entire application [4]. The entries are stored and the unique links between them are created inside the MySQL relational database management system. The communication between components is mediated by PHP programming language. The Apache is used as open-source web server solution. From software architecture point of view the database can be distinguished into two separate although mutually communicating layers. Namely these are the Data Access Layer (DAL) and Data Presentation Layer (DPL). The entire structure of the application and interactions between layers can be seen on Fig.1. Main development technologies and software solutions are listed in bold font. The entry search, create, read, update, and delete features of the Graphical User Interface (GUI) are summed up as SCRUD.

In total the REHABitation database is designed to store and spread information on exercises assessments tools and their inter connections but not to monitor usage and clinical progress or store related medical data of the users. The users are defined as all persons entering data, interconnecting entries or searching for them, including patients, device manufacturers, medical professionals and research staff. Users entering information into the database are responsible for the quality and content as they may only provide information based on their expertise which is ensured by the role registration process.

\subsection{Data Presentation Layer}

The DPL provides graphical user interface. The database entries are presented in clear and concise way in form of tables using a DatatTables plug-in for the jQuery Javascript library [9]. The user can search for exercises, assessments, equipment, systems and diagnosis separately however seeking of combinations is also possible. Focus of exercise or assessment lies within improvement of specific area. Information about the equipment or system is accessible by communication standards and may also obtains potential certification by recognized certification institutions. The ICD code identifies specific diagnosis, which is associated with an exercise or assessment 2. The filtered entries appear in real time, speeding up the entire process and also letting the user react on search result with each typed-in character. Visibility of columns and pagination (number of entries on one page) can be set by user as well.

A registered and authenticated user is also capable of creating, modifying or deleting entries via an additional graphical interface, which is inaccessible to the non-registered user. Entered information is validated against a set of basic rules on server side. The data validation routine checks whether all the mandatory fields are filled, text does not include special unrecognised characters outside of the typical UTF-8 character encoding and also prevents SQL injections attacks. In case the new entry does not comply with a rule the user is subsequently informed about expected data input format and asked to modify them accordingly. The new entry is created 
and published in the database on the assumption of rules adherence. Each entry is supplemented by the information about date of creation/modification and name of the user who created/modified it.

\subsection{Data Access Layer}

The (DAL) facilitates a connection and data fetching from the database to the DPL, where the user can view them. All the entries are stored on the server side and user does not interact with them directly. Taking a more detailed view on its structure the REHABitation database consists of several sub-databases:

- Exercise and Assessments sub-database associates information about name of the exercises or assessment together with concise description providing the overview about exercise expected outcome. The user is informed about approximate duration of the exercise and medical field it focuses. The sub-database also indicates whether the exercises are suitable for home-based or rather clinic-based rehabilitation and if the supervision is advisable. Potential restrictions and limitations that could be otherwise inconvenient for the patient performing the exercise are given in a separate field.

- Equipment sub-database lists available devices specifying each entry with the name of the equipment and description of its function. User can also access home-page of the device manufacturer. Further information provided differentiation between mobile and stationary equipment, whether the equipment is a physical device or a software tool and finally separate equipment into prototypes and released product categories. The information about connectivity options incorporated (Bluetooth, wifi, RS-232, Ethernet, USB) is also available.

- Diagnosis sub-database provides an overview of most common medical diagnosis linked with the field of rehabilitative medicine. Selected diagnosis are introduced by its name and uniquely specified by the International Classification of Diseases (ICD-10) code. The ICD is the standard diagnostic tool for epidemiology, health management and clinical purposes. It is owned and reviewed by The World Health Organization (WHO) and used in most countries (117) to report mortality data a primary indicator of health status. The ICD revision 11 is currently under development by WHO.[6]

- System sub-database connects together singular equipment components forming at the end entire systems which can take advantage of several devices working together usually simultaneously. For example Upper Extremity Therapy system encourages home-based rehabilitation in a form of simple games focused on increasing patient's range of motion.

The REHABitation database allows access to each of about mentioned sub-databases. In addition the main page of the database provides a comprehensive overview of the key information for each exercises in compressed format. Usage of modern web-development technologies, such as jQuery, Ajax and Bootstrap framework facilitates multiple features such as filtering of database entries in real time and responsive content of the web-page providing best user experience on commonly used devices (desktops, tablets and mobile phones).

The internal links between entries from the sub-databases and inclusion of novel exercises and rehabilitative concepts makes the REHABitation database unique in comparison to other available databases.

\subsection{User registration and authentication}

The non-registered user has access to all the entries stored in the REHABitation database as well as possibility to take advantage of all above described web-based application features. However, in order to ensure data integrity and forgo invalid or incomplete data insertions, only the registered and authentication user can submit new entries. Each registration request is reviewed by REHABitation project team keeping possible database intervention in a closed group of specialists.

The user can be assigned into the role of: Editor, Moderator or Administrator. The Editor can create a new database entry or modify an existing one. The Moderator confirms/declines changes of entries before they are stored into the database and can also delete them. Limitation of rights for specific sub-database for both above mentioned roles is also possible. The rights and roles are assigned by database administrator. [7].

Furthermore, separation of DPL and DAL prevents from insertion of potentially misleading information and also to a high extend protects the data already stored in the databased from being modified by an unauthorised third party. Automatic authentication requests from bots often try to overload server by log-in requests. In a worst case scenario the bots succeeds compromising the security of entire application. Standard security feature in a form of Completely $\mathrm{Au}$ tomated Public Turing Test To Tell Computers and Humans Apart (CAPTCHA) was therefore implemented.

\subsection{Practical Use Case - Stroke Patient}

In order to test and demonstrate capabilities of presented REHABitation database several use cases were adapted from rehabilitative scenarios encountered in real life. The planing of the clinical treatment is solely performed by medical professional, whereas the patient may use the REHABitation database for reassuring given information or retrieving additional information about the prescribed exercises/assessments and tools.

The medical specialist at the clinic is seeking for a rehabilitative exercise for a 73 years old female stroke patient. It is known that the patient lives together with her family which owns a Microsoft Kinect device. Patient's range of motion is in particular restricted in the left upper extremity. People affected by motion impairment tent to use the non-affected side for activities of daily living. Therefore, the rehabilitative sessions should be focused on retraining a precedent functionality and range of motion.

The patient and/or the medical specialist is looking for innovative exercises; new way of helping patient recover and retrieve range of motion after stroke. The REHABitation database is used as a intermittent tool suggesting viable exercises. On the one hand the medical specialist looks up innovative exercises; new ways of helping patients to recover and retrieve range of motion after a stroke. Searching in the database can be initiated by entering 




Figure 1: Overview of REHABitation Database Application. The Data Access Layer is separated into three main sub-databases and provides information about storage of entries and their internal links in the MySQL database. The Data Presentation Layer describes options mediated by Graphical user interface (Entry SCRUD and User authentication). The interface is based on Bootstrap and Datatables following principles of responsive web design

a keywords derived from the patient status. On the other hand a patient or his/her family can recreated a search result at home environment even simpler. Assuming the suitable exercise was suggested by specialised and performed by the patient at the clinic, its sufficient if the patient looks for name of the exercise even if its known only partially or other keywords.

\section{RESULTS}

Following a use case of the stroke patient the physician can browse the database by searching for suitable keywords such as: Stroke, Range of motion/Balance and Kinect. This search results in total of three exercises from which a two-phased clinically tested exercise named Bookshelf is chosen as a best fit [?].

\subsection{Detailed Entry View}

The detailed view of the Bookshelf exercise depicted on Fig. 3 together with its concise description, tips of how to perform it can be accessed by clicking on the search result. Such information, varying in content depending on the type and ownership, can be obtained for all exercises in the database.

Furthermore, footage of patient performing the exercise in the form video or image is presented under the detailed text information. This feature provides both patient and medical specialist with clear picture as to how the exercises should be performed. Thereby the inclusion of new exercises into the rehabilitative process is easier in comparison to situations when only a textual description is provided.

\subsection{Evolution of the Database}

The REHABitation database is a platform hosting exercises, assessments, equipment and systems used during rehabilitative processes. Evolvement of the number of entries contained in the database in each recorded category can be seen in Tab.1. From its launch the database hosted total of 240 entries and the ration between exercises \& assessments and equipment \& systems was almost 1:1. Further conducted research activities in 2017 coupled with new knowledge and sources increased the overall number of entries to 838. The most significant raise of 237(103) entries can be observed in the category exercises and assessments respectively.

The increase of database entries by more than a threefold underlines the rapid growth of the presented database in previous year and an active approach of the authorized users in supplying their expertise in a form of new database entries and interconnections.

Table 1: Number of entries in the REHABitation database based on month and year

\begin{tabular}{|c|c|c|}
\hline \multicolumn{3}{|c|}{ REHABitation Database } \\
\hline DB Entry & $10 / 2016-06 / 2017$ & $06 / 2017-02 / 2018$ \\
\hline Exercises & 67 & 304 \\
\hline Assessments & 43 & 146 \\
\hline Equipment \& Systems & 111 & 123 \\
\hline Diagnosis & 22 & 22 \\
\hline Total & 243 & 838 \\
\hline
\end{tabular}




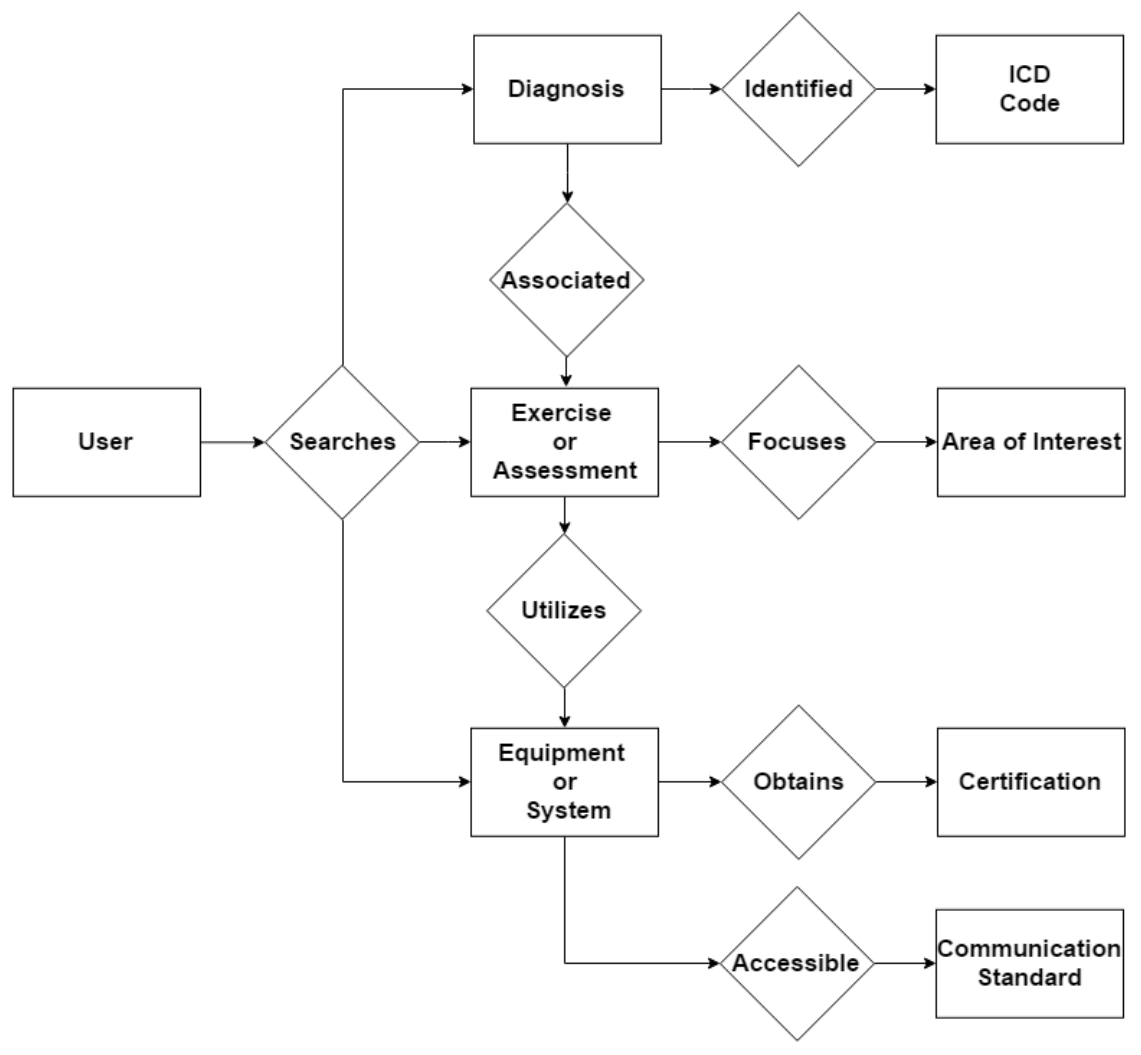

Figure 2: Overview of Presentation Layer. The User represents patient, physical therapist or technician looking for an information. There are several entry points. Exercises, assessments, equipment, system and diagnosis. Each entry point is clearly identified using standard (e.g. WiFi) or standardized labeling (e.g. ICD code). The user can choose and proceed the search from the individual point of view, that suits his needs best. Taken from [7]

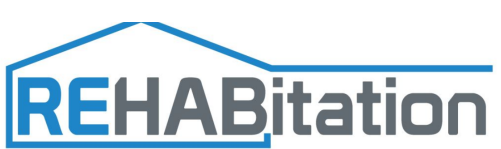

Detailed information about Bookshelf

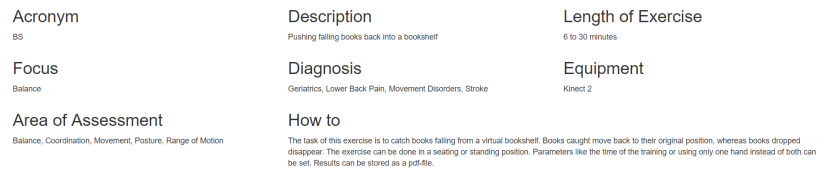

Figure 3: Detailed Entry View for Bookshelf rehabilitative exercise

\subsection{Worldwide Usage of the Database}

The results of continuous monitoring of REHABitation database utilizing Google Analytics Solutions is depicted on Fig.4. The overall number of people visiting the database in time period between 24.10.2016 - 26.2.2018 was 266 users (initiated at least one session). From the overall sum of active users a majority represents new visitor $(87 \%)$ and the remaining $13 \%$ were returning visitors. In total the database was accessed from 33 states. The largest margin of $79(28.94 \%)$ users comes from Austria. The United States and
United Kingdom with 27 (9.89\%) users and 26 (9.52\%) users hold second/third place, respectively. The users without set nationality were excluded from this statistic.

\section{CONCLUSION}

The REHABitation database provides information for the medical staff about which innovative or well established exercises/assessments they could include into the rehabilitation process. Information from each sub-database is reachable either separately or via the concise overview given on the main page. Medical specialists, patients or virtually any type of user can search through database entries in real time finding the required information quickly.

Authenticated user have extended privileges allowing them to not only search but also to add, modify, delete or link the entries. This complex network of internal links is specific for the presented database. Furthermore due to the internal links new combinations of exercises and equipment can be created. This approach is providing an option for inclusion of new exercises which can utilize novel tools such as Kinect, Wii balance board, smart phones and wearable sensors into the database. Additionally new developments with a high technology readiness level, like Sword Health [10] may be included and linked to corresponding exercises/assessments 


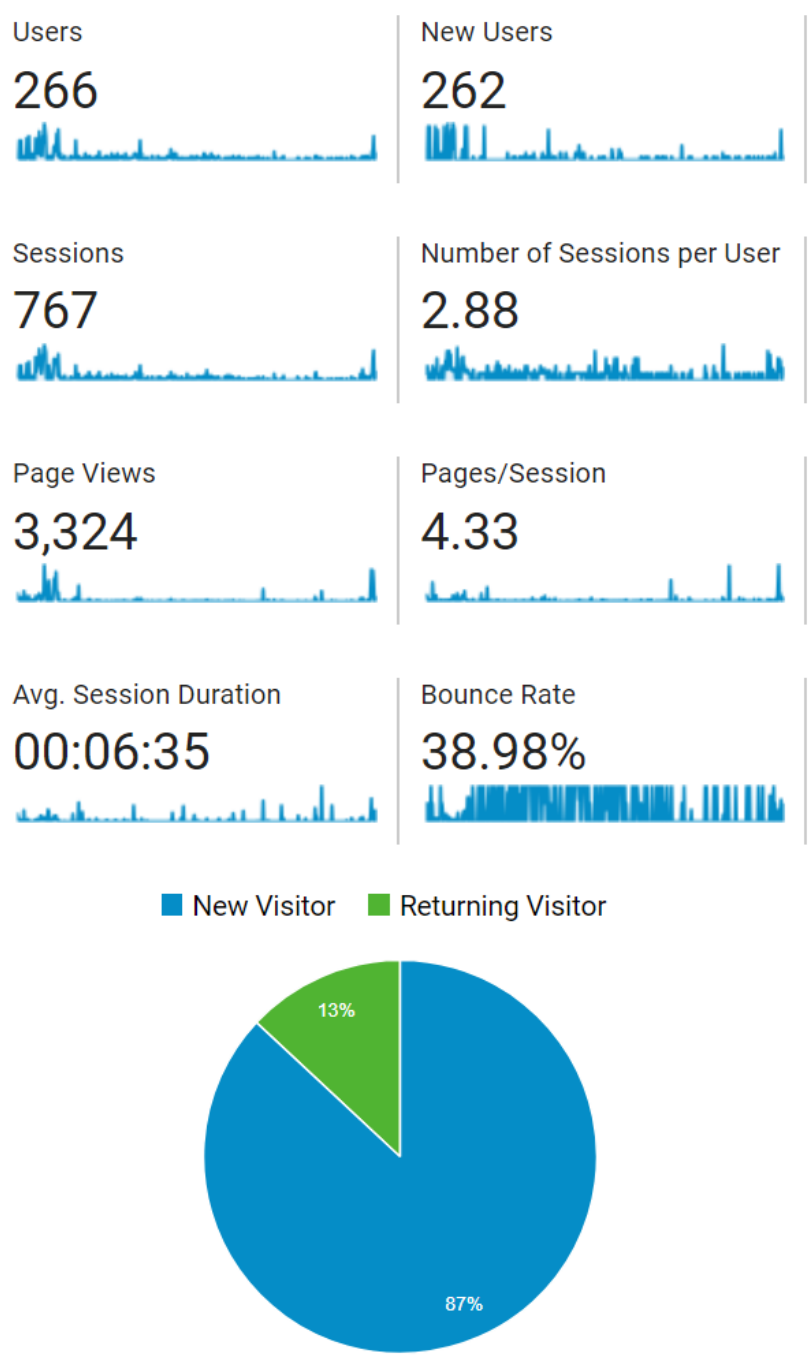

Figure 4: Overview of REHABitation database utilization in time period from 24.10 .2016 to 26.2.2018

and diagnosis. In addition insight into how-to effectively incorporate and utilize medical equipment already owned by the clinic for different exercises is also given. Hence potentially increasing effectiveness and number of exercises included in the rehabilitative processes. However, REHABitation database is acting as an information and collaboration platform and shall not replace existing tele-rehabilitation approaches. Moreover, the database is not designed to actually monitor the usage of the tools by the patient, their clinical progress or store any related medical data of the users.

The advancements related to further development and enrichment of the database over the course of research project REHABitation include: detailed information view about exercises, practical how-to suggestion for performing the exercise in form of textual description and incorporation of media files such as images or embedded video recordings featuring patient or staff while practising the exercise. The changes reflect suggestions collected during workshops as well as feedback obtained from users within the REHABitation team who actively used the database over extended period of time.

Future challenges include accessibility tests and adoptions for the focused user groups according to gold standards and W3C conventions. Additionally to the implemented ICD codes the rehabilitation specific ICF (The International Classification of Functioning, Disability and Health) coding convention will be implemented to align the usability of the database with the known workflow from the professional environment. Even though the database was developed under the principle of privacy by design, the current requirements given by the new General Data Protection Regulations of the EU still need to be addressed.

\section{ACKNOWLEDGMENTS}

The authors thank the clinical partners from the Sophienspital and NRZ Rosenhügel as well as all colleagues from the REHABitation project of the UAS Technikum Wien for their support during the research process. The project REHABitation is funded by the Austrian Research Promotion Agency FFG.

\section{REFERENCES}

[1] Richard J Cook, Katherine Berg, Ker Ai Lee, Jeffrey W Poss, John P Hirdes, and Paul Stolee. 2013. Rehabilitation in home care is associated with functional improvement and preferred discharge. Archives of Physical Medicine and Rehabilitation 94, 6 (2013), 1038-1047. https://doi.org/10.1016/j.apmr.2012.12.024

[2] Carlos Faria, Jorge Silva, and Aurélio Campilho. 2015. Rehab@home: a tool for home-based motor function rehabilitation. Disability and Rehabilitation: Assistive Technology 10, 1 (2015), 67-74. https://doi.org/10.3109/17483107.2013.839749

[3] Google. 2018. Google Analytics Solutions - Web Analytics \& Marketing Measurement - Google. https://www.google.com/analytics/

[4] James Lee and Brent Ware. 2002. Open Source Development with LAMP: Using Linux, Apache, MySQL and PHP. Addison-Wesley Longman Publishing Co., Inc., Boston, MA, USA.

[5] R Müller and A Klimesch. 2011. Entwicklung der ambulanten medizinischen Rehabilitation in Österreich. In Reha-Bericht 2011. Pensionsversicherungsanstalt, Vienna, 26-30.

[6] World Health Organization. 2018. WHO | International Classification of Diseases. http://www.who.int/classifications/icd/en/

[7] Richard Paštěka and Mathias Forjan. 2017. Development of a Multidisciplinary and Telemedicine Focused System Database. In Studies in Health Technologies and Informatics, Vol. 236. IOS Press, 144 - 151. https://doi.org/10.3233/ 978-1-61499-759-7-144

[8] PeThe Hrvatska. 2018. PeThe - Personal Therapist System. http://pethe.eu/

[9] SpryMedia Ltd. 2018. DataTables | Table plug-in for jQuery. https://datatables. net/

[10] SWORD Health. 2016. SWORD Health: Reinventing Physical Therapy. https: //www.swordhealth.com/

[11] World Health Organization. 2011. WHO / World report on disability. Technical Report. http://www.who.int/disabilities/world\{_\}report/2011/en/

[12] Lena Zidén, Margareta Kreuter, and Kerstin Frändin. 2010. Long-term effects of home rehabilitation after hip fracture 1-year follow-up of functioning, balance confidence, and health-related quality of life in elderly people. Disability and Rehabilitation 32, 1 (2010), 18-32. https://doi.org/10.3109/09638280902980910 